\title{
Support system for the training of crisis management group members
}

\author{
M. Drozdova ${ }^{1}$, P. Rapant ${ }^{1} \&$ L. Malerova ${ }^{2}$ \\ ${ }^{I}$ Department of Geoinformatics, VSB - Technical University of Ostrava, \\ Czech Republic \\ ${ }^{2}$ Department of Civil Protection, VSB - Technical University of Ostrava, \\ Czech Republic
}

\begin{abstract}
A unique training workplace for public service employees, who deal with extensive crisis events, is now being prepared in the Czech Republic. The Czech Republic has suffered several floods in the recent years, floods are therefore the principal concern, but other events such as dangerous chemical leaks or extensive traffic accidents are taken into account. Despite being inspired by similar workplaces in the neighboring countries, the proposed solution remains unique. The output of this project will be a piece of specialized software, which will constitute a simulation environment over the GIS projects, and a set of workplaces. These workplaces will enable the course participants to be exposed to situations similar to real life situations, which might occur during unusual events. The project is being prepared with the support of the Ministry of Interior of the Czech Republic within a research project concerning public safety called SIMPROKIM - Crisis Management Process Simulator and with the support of the Fire Brigade of the Czech Republic, represented by the Czech Association of Fire Officers. The first stage was focused on tools and methods of crisis management process specification and a solution as to how to create a system, which would support training of the emergency committee members. Business modeling methods were used for the specification of crisis management processes and the processes of the emergency committee member training. Also Unified Software Development Process was used to design a system that will support the emergency committee members training. System requirements were elicited and described in the System Requirements Specification Document, which was passed to a company that was responsible for the project
\end{abstract}


implementation. Selected tools and methods were proven to be suitable, because they supported the communication among experts from various fields properly (crisis management, geoinformatics, software engineering). The authors have also designed a subsystem for creating training scenarios, which is used for the preparation of scenarios for every training exercise.

Keywords: crisis management, emergency, simulation software, training scenario.

\section{Introduction}

People must face the consequences of various events which cause damage to property and human health and increase death toll. Among these, there is a group of events that cannot be dealt with by the common integrated rescue system routine, i.e. the units foreordained to solve these events (Fire and Rescue Brigades, Police of the Czech Republic). Multistage systems of crisis management bodies are being formed for these extensive events and crisis situations. These represents the management segment of crisis management, while the integrated rescue system forms the executive segment.

The activities of crisis management bodies are specified mostly by the Czech Republic legislation. Integrated methodic procedures for the fighting of specific crisis situations are defined only to a limited extent. Major problem is the issue of systematic training of members of crisis management bodies for their performance in overcoming crisis situations.

In 2010, the Faculty of Safety Engineering at the VSB - Technical University of Ostrava (FBI VŠB-TUO) commenced work on the SIMPROKIM (Simulation of crisis management processes in the system of whole-life education of the integrated rescue system (IRS) units and public administration bodies) project. The SIMPROKIM project falls under the framework of Safety research program, financed by the Ministry of Interior of the Czech Republic (MI CR), for which two main objectives have been determined:

1. To prepare the specification of crisis management processes for selected crisis situations in the form of obligatory methods accepted by MI CR;

2. To create the support system for the systematic training of permanent crisis management group members at the level of municipalities with extended competence (MEC), including its software that provides regular training of crisis management group members for fighting selected crisis situations.

Nowadays, there are 70 types of emergencies defined in the Czech Republic that can lead to the crisis situation. Crisis situation is legally as an extensive event with ,harmful effect of forces and phenomena produced by human activities and natural effects, as well as the accidents that endanger lives, health, properties or environment and require rescue and liquidation activities." ( $\$ 2$ of the IRS act) [1]. Within the SIMPROKIM project scope, the researchers have committed to elaborate five most frequent crisis situations. The following crisis situations have been selected in the cooperation with other subjects:

- flood,

- traffic accident, 
- forest fire,

- leak of dangerous substrate,

- fire of industrial object or waste dump.

This article is focused on the specification of the requirements and analysis of the software system supporting the training of permanent crisis management group members at the level of municipalities with extended competence. Especially it focuses on creating individual scenarios and principles of system support during the actual training exercise.

\section{Conception}

The whole solution must be independent on specific crisis management processes during crisis situation fighting and must, in the future, enable the modification of present processes and definition of new crisis management processes for any other crisis situation.

\subsection{Training center}

The training workplace conceptually consists of several sections: the workplace of permanent crisis management group, the workplace of communication with media and the workplace of direction. The whole training system is designed to enable training realization either in regular spaces established for this purpose, or in the premises provided by the subject whose employees will be trained. Several technological requirements had to be secured in order to assure mobility of the training center. Among these requirements are data transfer between server and mobile computer workstations, IP based telephony system, and mobile center supporting multimedia communication.

\subsection{Software system}

Software system supporting the training of crisis management group members has two main functions: simulate the course of crisis situation and support the activities of crisis management group that the group must perform in order to fight the crisis situation effectively. The system support is available during the whole organization of training and educational process, from the course announcement to the registration of participants, to creating their accounts, to final issuing of certificates on passing the course.

\subsection{Chronological and geographical aspects}

The training of crisis situation fighting runs in two time lines simultaneously. The first time line deals with emergency that has its characteristic course corresponding to the real crisis situation development in given territory (operational time). The second time line represents the time needed for the actual training (astronomical time). Real life crisis situation itself develops in the time range of days to weeks. Generally, it is impossible to meet such a period of time 
during the training, therefore it is necessary to establish the principles and mechanisms of time leaps within the training exercise.

Crisis situations and their fighting proceed not only in time but in space as well. The support system for training must therefore utilize the means of Geographic Information Systems (GIS). GIS is used to display and register information related to crisis situation and to display prepared maps, which correspond to the given phase of crisis situation development in the actual operational time.

\section{Requirements engineering}

The SIMPROKIM project aims to develop an automated system that will supply necessary methodological, technical, programming and data environments. These environments will provide training in the field of crisis management, public administration and the IRS. Training will be provided by resolution of the security situation in the area in three basic tasks: protection, response and recovery. Individual tasks are further divided into stage of education, training and testing. Training should offer option to prepare either individuals or all members of team.

\subsection{Procedures}

The first phase of the project focuses on the identification and description of crisis management processes. Processes are modeled using the principle of business modeling. Crisis management process (CMP) is an ordered set of activities that collectively reach the strategic goal - overcoming crisis situation. In the context of the organizational structure, CMP defines roles of crisis management bodies, their functions and relationships [2]. CMP are modeled using activity diagram specified in Unified Modeling Language (UML) [3].

Rational Unified Process (RUP) methodology was used in order to specify and analyze requirements efficiently and in well-known and unified manner. The Rational Unified Process is an iterative software development process framework, which covers project whole life cycle. The requirements are usually dynamic and it is necessary to keep in mind the fact that requirements could change in the future. This is demonstrated by IKIWISI "I Will Know It When I See It" syndrome [4], that expresses the inability to specify all the requirements of the intended system in advance. It is therefore necessary to manage requirements, which is arranged by the use of a Software Requirements Specification (SRS) document that is being versioned with every requirements change.

\subsection{Reference task}

The implementation of each phase of training tasks is conditioned by playing pre-created, strictly defined scenarios. Response task was identified as most dynamic and complex task, that deals with Response task in the specific area. This task simulates the course of crisis situations which activities defined in the 
crisis management process are closely related to. This task was due to its specific character selected as a reference when designing the mechanism of the scenario and its subsequent playback.

When analyzing the requirements for this task general set of activities was defined together with the process how to undertake these activities by a group of course participants, which reflects the composition, powers and duties of crisis management group (CMG). Basic working processes of $\mathrm{CMG}$ carried out during the course (shown schematically in figure 1), are defined as follows.

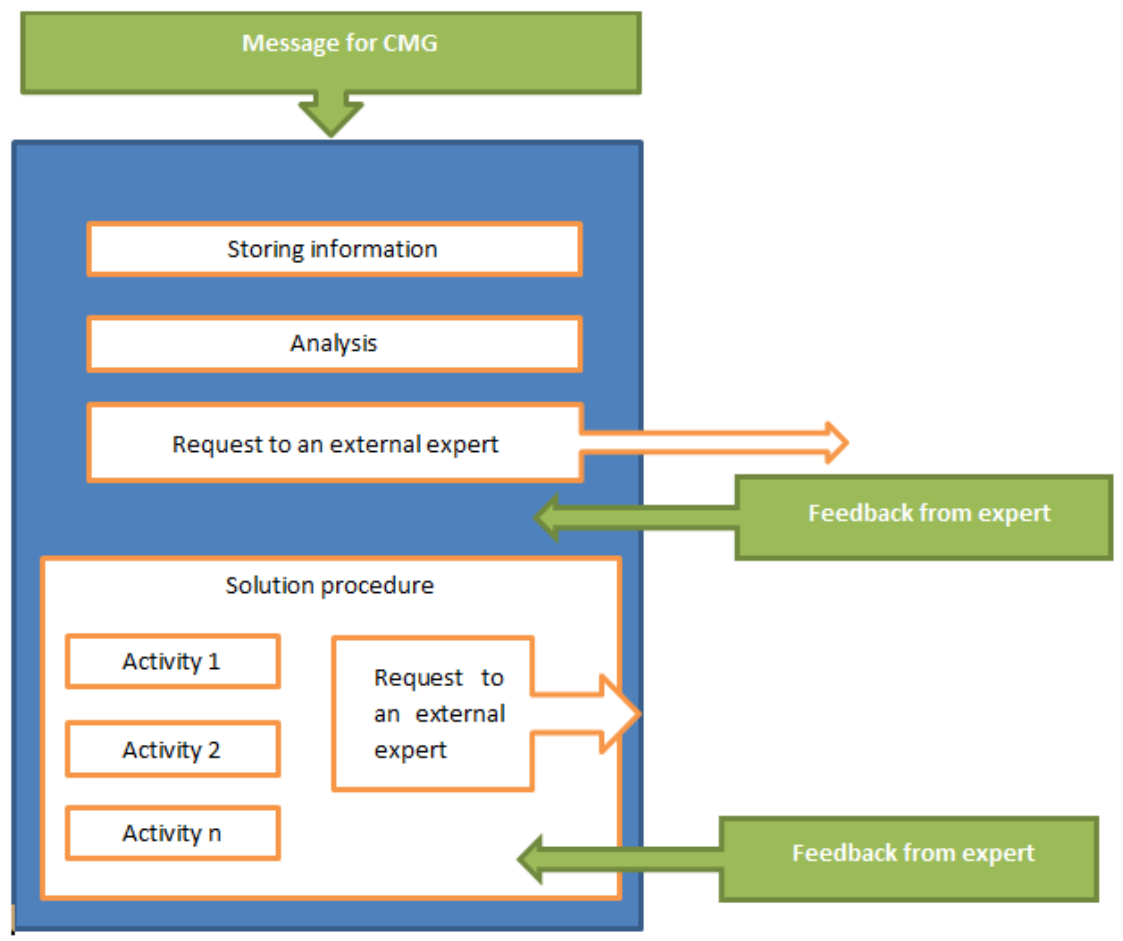

Figure 1: $\quad$ Basic working processes of $\mathrm{CMG}$.

CMG receives a message, stores information about this message and analyses it. Assessing the situation and a decision on the most appropriate measures, however, is not the responsibility of the CMG. CMG is therefore forced to send a request to an external expert that provides $\mathrm{CMG}$ with feedback about the best nature and location of the measure. CMG meanwhile conducts its own partial activities of their work, during which they can send requests to experts.

Message can represent reports about spilling any water flow. Feedback delivered to the CMG from external expert may contain the information on the most suitable site for the construction of a temporary dam and its character. Feedbacks from experts are prepared in advance and they are included in the course scenario. 


\subsection{Summary of key facts}

The whole software system is based on the requirements analysis divided into three parts: a public portal, subsystem for the creation and playback of scenario exercises and a part that contains modules that are needed for the overhead required to complete the organization of the educational process.

Geographic information systems are used to simulate emergencies by displaying a sequence of time slices of predefined emergency in the territory. All the measures addressed in crisis management activities are also prepared in advance and incorporated into the scenario.

The system will be able to monitor and record all activities of trainees continuously, allowing continuous evaluation of the trainees and members of permanent working groups.

\section{Subsystem of training scenario creation}

Both in theory and software system, creating scenarios is a very demanding activity that requires concentration and precision from scenario-writer that creates the scenarios. Scenario creation mechanism is designed to facilitate the user's (scenario-writer) work as much as possible when converting theoretical ideas that capture the principle of scenarios into schematized form inside the system environment. The elements of scenarios and their structure as well as a module for creating scenarios as a whole are designed in the way to simplify the process of scenario creation. This design also supports high level of scenario process automation during the course.

The module for creating scenarios therefore represents environment that provides resources and functions for creating training scenarios.

\subsection{Scenario}

Each course follows relevant scenario, whose length must match the planned duration of the course. The aim of the scenario is to represent the issues taught. These issues are artificially created emergencies and the process of dealing with them is controlled by sequence of predefined steps. When following these steps trainee is forced to use the methodology of crisis management.

Structure of the scenario captured in figure 2, represents various measures that can be dealt with within selected emergencies. The scenario is a set of events that in most cases reflects individual measures that the trainee must deal with during the course. An event is a set of activities designed by a scenario-writer so that its sequence realizes a course of event. An activity is a fundamental element of the scenario.

\subsection{Activity}

The course of events, that the scenario is composed of, is realized by a sequence of activities. Each activity is described by a set of base and complementary parameters that are assigned with the activity on the basis of its thematic 


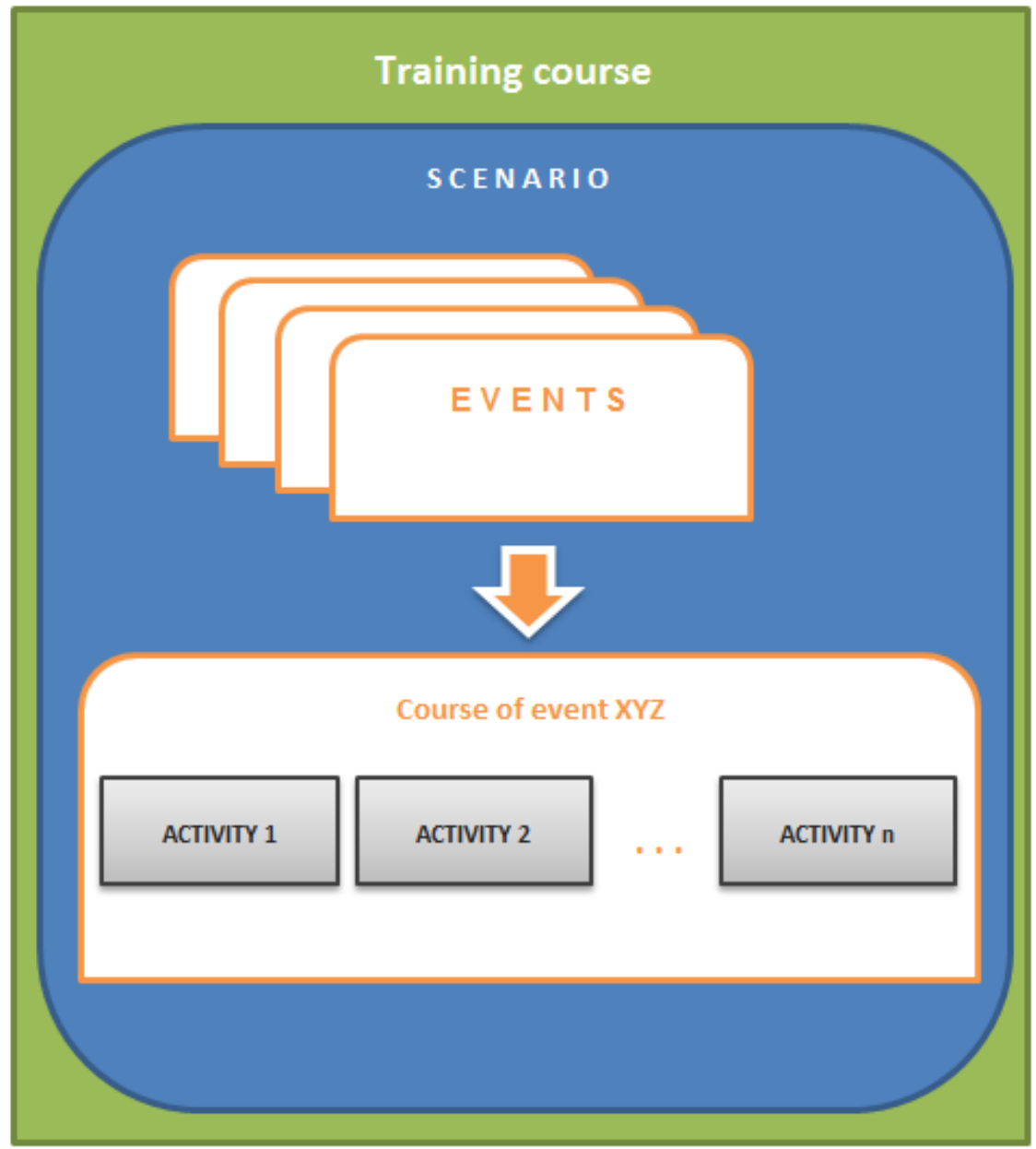

Figure 2: $\quad$ Schematic arrangement of individual elements in scenarios.

classification. The base parameters are: title, actor, type and content. The classification of activities is determined by combining the values of the type and actor parameters. An actor is a name for an entity that carries out the activity. In the context of the described system the parameter acquires the following values: system, simulation, permanent working group and others. A type indicates a specific nature of the activity, depending on the value of the actor parameter. Values of complementary parameters determine how the activity will be carried out. The matrix schematically shown in figure 3 is a possible representation of assignment of additional parameters to activities that is determined by combinations of the actor and type parameter values. 


\begin{tabular}{|c|c|c|c|c|c|c|c|c|c|c|c|c|}
\hline & \multicolumn{3}{|c|}{ SIMULATION } & \multicolumn{3}{|c|}{ SYSTEM } & \multicolumn{3}{|c|}{ SPS } & \multicolumn{3}{|c|}{ OTHERS } \\
\hline & 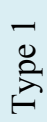 & 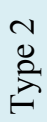 & 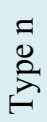 & 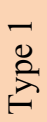 & 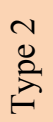 & 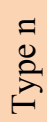 & 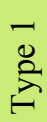 & 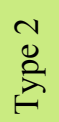 & ह & $\overrightarrow{0}$ & 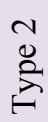 & 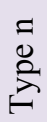 \\
\hline Parameter 1 & & $\mathrm{x}$ & $\mathrm{x}$ & & & $\mathrm{x}$ & & & $\mathrm{x}$ & & & \\
\hline Parameter 2 & & & & & & $\mathrm{x}$ & $\mathrm{x}$ & $\mathrm{x}$ & $\mathrm{x}$ & $\mathrm{x}$ & $\mathrm{x}$ & \\
\hline$\ldots$ & $\mathrm{x}$ & & & & & & & $\mathrm{x}$ & $\mathrm{x}$ & & & $\mathrm{x}$ \\
\hline$\ldots$ & $\mathrm{x}$ & $\mathrm{x}$ & & & $\mathrm{x}$ & & $\mathrm{x}$ & $\mathrm{x}$ & $\mathrm{X}$ & & & \\
\hline Parameter $\mathrm{n}$ & $\mathrm{x}$ & & & $\mathrm{x}$ & & & & & & & $\mathrm{x}$ & \\
\hline
\end{tabular}

Figure 3: $\quad$ Complementary parameters matrix.

\subsection{Event and its course}

An event is a set of activities designed by a scenario-writer so that its sequence realized thematically the intended course of events. Each event is described by the following set of parameters: name, entity responsible for an event, scope, description, address, coordinates.

The course of events is implemented by a sequence of activities. Each activity from an order set of activities is assigned with a set of reference activities and their statuses, which determines the position of an activity in the event. This mechanism therefore creates a timeline of an event that the individual activities are suspended to.

When creating the course of event, the drag and drop technique enables to add pre-prepared activities to the timeline or to create a new activity. The position of each activity inside the event is described by following parameters: reference state, reference activity and delay. The event as a whole always has a determined duration that reflects set of activities, however this duration is being continuously recalculated when the activity is executed.

A reference activity is an activity from whose reference state parameter, the launch of next activity is calculated. The activity can be in one of these reference states: initialized, terminated, executed. The value of the delay parameter determines position in time of a particular activity in the sequence of activities throughout the event.

\subsection{Scenario process}

The scenario covers the content of the entire lesson. It consists of a set of events that must be already prepared. Events do not have to be included in the scenario in sequential succession. Partial or complete parallel concurrence of two or more events is allowed and expected. This situation is shown in figure 4. 


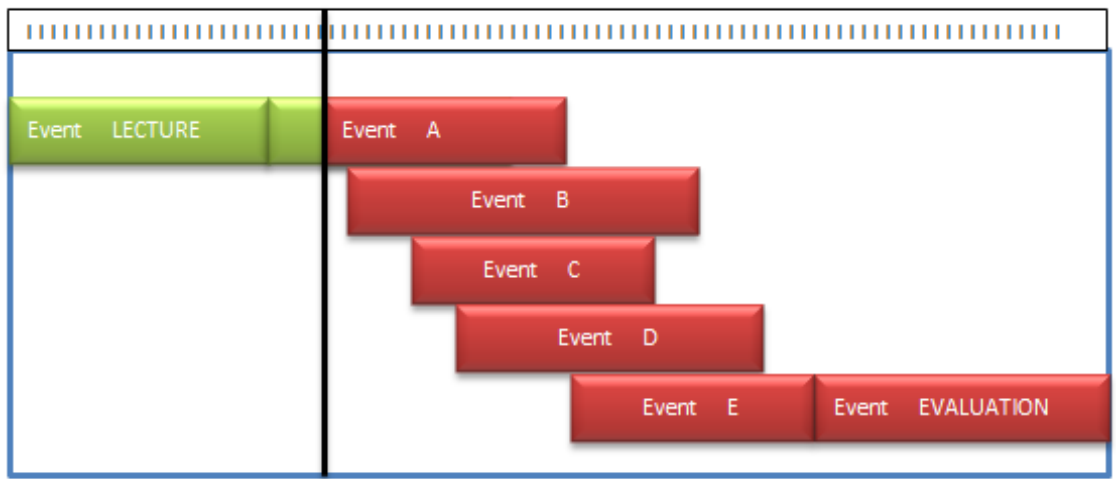

Figure 4: Queue of events during the course of the scenario.

The scenario-writer can assign the bodies and resources to each scenario. CMG respectively crisis management group members may manage these resources as they consider it appropriate. List of bodies and resources can have any number of records. A record consists of an item selected from the code of bodies and resources and a figure meaning the amount available. Deployment of the bodies and resources is based on crisis management plan of the target territory, where the simulated emergency is situated.

A necessary task when creating scenarios is the time initialization of an emergency, for example: 00:00 $=20.7 .2010$ 9:00. This triggers the initial synchronization of the operating time with the astronomical time of the course.

Application logic of playing the scenario is based on the importance of individual activities and values of their parameters. Through these values sectional system functions are defined. Their gradual execution realizes the playing of individual activities included in the events and therefore realizes the playing of the whole scenario.

\section{Working environment}

In order to execute courses with the maximum use of system support, it is necessary for the realization of the scenario to create a working. This is an environment, where a trainee performs activities within his set of responsibilities. Environment should be designed to enable the trainees to respond to impulses of the simulated situation that come from the scenario director.

Specification of the working environment for each role of CMG reflects a set of activities, which the CMG members are responsible for. Each trainee will be logged into the system with a specific role. The system will set up a role-specific working environment. A trainee is assigned to the role using a system component - course organization agenda.

The following working environments were defined within the system: journal environment for incoming and outgoing messages, situational journal 
environment, environment for proposed report forms for crisis management group, environment for proposed crisis management group chairman decision, bodies and resources evidence environment, available bodies and resources evidence environment, environment for recording information acquired by the representatives of the IRS, environment for creating public relations reports, GIS environment- situation map and additional environments such as mail client, phonebook, document library and read only GIS.

\section{Conclusion}

The aim of the SIMPROKIM project is to solve two problematic areas of the crisis management within the Czech Republic. First, there is no unified specification of the crisis management processes when dealing with different types of emergencies. Secondly, there is an absence of systematic crisis management training of permanent crisis management group members at the level of municipalities with extended competence.

This article focuses on the software system that aims to automate regular training of the members of crisis management group based on coping with artificially simulated emergencies. The main part of this system is a module for creating scenarios on the basis of which the individual training courses are realized. The scenario covers both simulation of emergency and ongoing activities within the permanent crisis management group, whose members participate in courses.

The article describes the process of requirements specification. The mechanism of creating training scenario is presented and discussed in detail on the basis of a requirements analysis. Steps in the project are regularly consulted with the representatives of crisis management group and members of the Czech Association of Fire Officers so that the resulting system covers the needs and demands emerging in real crisis management.

\section{Acknowledgement}

This research is supported by the grant no. VG20102015043 "Simulation of crisis management processes in the system of lifelong education of IRS units and public administration bodies" in the framework of the Safety Research program of MI CR.

\section{References}

[1] Act no. 239/2000 Sb., of integrated rescue system and of the amendments of some laws, as amended by act no. 320/2002 Sb.

[2] Vondrák I.: Metody byznys modelování (Methods of Business modeling), VŠB-TUO, 2004.

[3] Specification UML 2.0. http://www.omg.org/spec/UML/2.0/

[4] Boehm B.: Requirements that Handle IKIWISI, COTS, and Rapid Change, Computer, 33(7), pp. 99-102, July 2000. 